\title{
Editorial
}

\section{A Balancing Act: The Medicare Audiologist Access and Services Act of 2019 and the Treatment of Dizzy Patients}

DOI: $10.3766 /$ jaaa.31.3.1

$\mathrm{T}$ he Medicare Audiologist Access and Services Act of 2019 (H.R. 4056) is bipartisan legislation introduced into the U.S. House of Representatives on July 25, 2019, by Reps. Tom Rice (R-SC), Matt Cartwright (D-PA), Mark Meadows (R-NC), Ralph Norman (R-SC), Mike Kelly (R-PA), Jan Schakowsky (D-IL), Brad Schneider (D-IL), Ann Kuster (D-NH), Gus Bilirakis (R-FL), and Lisa Blunt Rochester (D-DE). The bill, if passed, would enhance federal benefits for hearing and vestibular/balance health by providing Medicare beneficiaries direct access to both diagnostic and therapeutic services provided by audiologists.

Specifically, this legislation would: 1) give audiologists additional opportunities under Medicare with reclassification as "practitioners," 2) allow for direct access by Medicare patients to audiologists, and 3) expand covered audiological services beyond diagnosis to include treatment. It is exciting that our three largest national organizations representing audiology-the American Speech-Language-Hearing Association (ASHA), the American Academy of Audiology (the Academy) and the Academy of Doctors of Audiology (ADA) - came together to collaboratively endorse this bill.

The opportunity for patients with Medicare to directly access audiologists for diagnostic and treatment services would certainly improve hearing health care for these beneficiaries. There are also, however, many audiologists who assess and treat patients with balance disorders. In fact, 24 percent of the membership of the American Academy of Audiology identify themselves as routinely performing vestibular/balance assessments.

\section{THE AUDIOLOGY SCOPE OF PRACTICE}

$\mathrm{T}$ he diagnostic activities (e.g., caloric testing and rotational testing) associated with vestibular and balance assessment have specific CPT codes and documented national payment rates. For both the Academy and ASHA, the assessment and treatment of vestibular/balance disorders is also clearly stated as being within audiologists' scope of practice (SOP).

The Academy's SOP states that: "Audiologists also are involved in the treatment of persons with vestibular disorders. They participate as full members of balance treatment teams to recommend and carry out treatment and rehabilitation of impairments of vestibular function." Similarly, the ASHA SOP states that: "If the audiologist can document appropriate training for new and emerging clinical or technological procedures that fall under the heading of auditory, balance and other related disorders, then such innovations and advances may be incorporated into the Audiology Scope of Practice."

Therefore, since vestibular/balance treatment clearly falls within the audiology SOP and, if H.R. 4056 passes, this should allow/encourage appropriately educated and trained audiologists to perform (and be reimbursed by Medicare) for vestibular rehabilitation.

One question to consider is that, with the successful passage of H.R. 4056, will academic audiology programs need to adjust for these national changes, or are students already being appropriately educated and trained to deliver vestibular rehabilitation?

The Accreditation Commission for Audiology Education (ACAE) and the Council on Academic Accreditation (CAA) are the two bodies that accredit graduate programs that prepare individuals to enter professional practice in audiology.

The Diagnosis and Management section in the Curriculum Standards of the ACAE standards from 2016 states that students from the institution will be able to:

“(1) Diagnose, triage, treat, and manage auditory vestibular/balance conditions and disease for patients over the lifespan..." 
Likewise, curriculum standards from CAA (Section 3.1.6A) state that students will be able to:

"-perform assessment for vestibular rehabilitation;

-develop and implement treatment plans using appropriate data..."

So, it appears that our scope of practice encompasses audiologists engaging in assessment, as well as the treatment, of those with vestibular/balance disorders. There is also alignment among our relevant accreditation-body requirements and scope-of-practice documents regarding treating patients with balance impairments.

\section{EDUCATION AND TRAINING STANDARDS}

$\mathrm{H}$ owever, with only a quarter (reportedly) of audiologists engaged in the assessment and treatment of those with vestibular/balance disorders, this seems like a good time to ask our accrediting bodies to review and possibly update the minimal education and training standards specifically related to performing vestibular rehabilitation. Perhaps, as for physical therapists, a subspecialty certification (or perhaps a micro-credential) in vestibular/balance could eventually be developed for audiologists by our national organizations.

If you do not feel like you are adequately educated or trained to perform vestibular rehabilitation, then, of course, you should not do so (remember, regardless of how it is worded in our code of ethics documents: Do No Harm). Short courses are available specifically to educate/train audiologists, physical therapists, and others to enhance their knowledge of both the diagnosis and treatment of those with vestibular/balance disorders.
Our professional meetings frequently have presentations that focus on the vestibular/balance part of our SOP, so attend a few of them. You could also arrange to work with a colleague with expertise in this unique aspect of the field of audiology until you are comfortable performing these vestibular/balance services. Our audiology training programs should be preparing us to become lifelong learners-and this is an area that has evolved since many of us graduated and became audiologists.

\section{A NEW ERA IN AUDIOLOGY}

$\mathrm{T}$ his increasing collaboration and coordination among our major professional organizations to move legislation forward that would allow us to practice at the top of our license represents a new era in audiology. The fact that we have been largely viewed by payers as only diagnosticians has limited our ability to use to the full extent the rehabilitative services we have to offer our patients.

There is no question that the passage of H.R. 4056 would allow audiologists to be more consistently compensated for therapeutic services, but we must ensure that we are adequately prepared.

Devin L. McCaslin, PhD Deputy Editor-in-Chief

Robert F. Burkard, PhD

Neil T. Shepard, PhD 